\title{
Comparison of endoscopic and microscopic tympanoplasty in patients with chronic otitis media
}

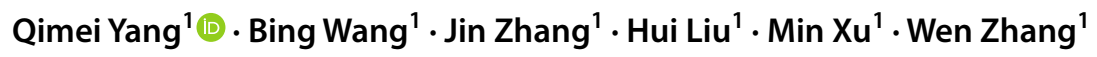

Received: 15 November 2021 / Accepted: 13 January 2022 / Published online: 5 February 2022

(c) The Author(s) 2022

\begin{abstract}
Background To compare the results between type I tympanoplasty performed with transcanal endoscopic ear surgery (TEES) and microscopic ear surgery (MES) for treatment of chronic otitis media in a homogenous group of patients.

Methods A retrospective study was performed in our department between January 2011 and January 2016 to review primary type I tympanoplasty cases with a subtotal perforation, an intact ossicular chain, a dry ear for at least 1 month, normal middle ear mucosa, and a follow-up period of at least 6 months post surgery. The adoption of TEES or MES was divided temporally (before and since 2013). TEES was undertaken in 224 patients (224 ears) and MES in 121 patients (121 ears).

Results The successful graft take rate was $94.64 \%$ (212/224) in the TEES group and 90.91\% (110/121) in the MES group $(P=0.239)$. The improvements in the air conduction levels between the 2 groups were not statistically different at 1,3 , or 6 $(>6)$ months $(P>0.05)$ after surgery. The improvements in the air-bone gaps were not significantly different between the 2 groups $(P>0.05$ ). The average hearing gains in the TEES group 6 (and $>6$ ) months post surgery were $11.85 \pm 5.47 \mathrm{~dB}$, which was better than $10.48 \pm 5.18 \mathrm{~dB}$ in the MES group $(P=0.031)$. The use of medical resources was lower in the TEES group than in the MES group regarding the average operating time $(49.22 \pm 8.24 \mathrm{~min}$ vs $81.22 \pm 14.73 \mathrm{~min}$, respectively; $P<0.0001$ ). Patients receiving MES had a significant higher incidence of the wound problems (ear pain, numbness around the ears, and wet ear; $P<0.05$ ).
\end{abstract}

Conclusion TEES for type I tympanoplasty seems to achieve a shorter operative time and ideal tympanic membrane healing rate and hearing results in patients with chronic otitis media.

Keywords Endoscope ear surgery $\cdot$ Microscopic $\cdot$ Tympanoplasty $\cdot$ Chronic otitis media

\section{Abbreviations}

PTA Pure tone average

ABG Air-bone gap

95\% CI 95\% Confidence interval

TEES Transcanal endoscopic ear surgery

MES Microscopic ear surgery

COM Chronic otitis media

\section{Introduction}

Type I tympanoplasty, or myringoplasty, is an effective measure to repair the tympanic membrane and enable the recovery of hearing loss in case of chronic otitis media [1]. It is typically performed via microscopic ear surgery (MES) worldwide [2]. The microscope equipment offers binocular vision along with an excellent stereoscopic surgical view and leaves both the surgeon's hands free, but it is limited by the straight-line vision that makes visualization of the middle ear through the ear canal relatively difficult. The microscopic surgery requires an upside-down conical "working space," resulting in much more soft tissue resection, with or without drilling of the bone to obtain adequate illumination [3].

Since the late 1990s, some physicians have successfully used endoscopes instead of microscopes for partial ear surgery [4]. The endoscope for middle ear surgery has been a highly contentious subject with the introduction of highquality narrow-diameter endoscopes, ultra-high-definition
Wen Zhang

smileww@foxmail.com

1 Department of Otorhinolaryngology-Head and Neck Surgery, Shaanxi Provincial People's Hospital, Xi' an 710068 , China 
cameras and screens, and cold light sources [5]. In contrast to the microscope, the endoscope has a cone-shaped source of illumination, which ensures optimal visualization and allows a wider field of view of the surgical area [6]. Endoscopic ear surgery permits a wide-angle view throughout the external ear canal, including magnification of the middle ear structures and direct visualization of hidden areas [7], such as the sinus tympani, hypotympanum, posterior part of the mesotympanum, and epitympanum [8].

In this retrospective study, we compared the results of type I tympanoplasty performed with transcanal endoscopic ear surgery (TEES) versus MES for treatment of chronic otitis media with a subtotal perforation, an intact ossicular chain, a dry ear for at least 1 month, and normal middle ear mucosa, over a postsurgical follow-up period of at least 6 months. We then evaluated whether the endoscopic approach offered advantages over conventional surgery.

\section{Materials and methods}

\section{Study designs}

This retrospective study was performed to assess functional results in patients with chronic otitis media who had undergone type I tympanoplasty at Shaanxi Provincial People's Hospital between January 2011 and January 2016. This study was approved by the Institutional Review Board of the Shaanxi Provincial People's Hospital.

A total of 682 patients underwent primary tympanoplasty during this period, with all surgeries having been performed by experienced otologist (W. Zhang). The study included a homogeneous group of patients with a subtotal perforation, a dry ear for at least 1 month, an intact ossicular chain, and normal middle-ear mucosa.

We excluded from the study patients younger than 18 years of age; those requiring ossiculoplasty, atelectasis or mastoidectomy; those with obvious narrowing of the external ear canal or syndromes that can affect the middle ear (e.g., Down syndrome); and those with a history of previous otological surgery.

A total of 345 patients were enrolled in the study. Patients were divided into 2 groups based on whether they received TEES (224 ears, 224 patients) or conventional MES (121 ears, 121 patients). The use of MES or TEES had a clear temporal division (before 2013 versus 2013 and after). As of January 2013, TEES was the primary procedure, and MES was used as a salvage technique in cases in which TEES was not suitable, such as in patients with ear canal stenosis.

All surgical procedures were performed by the senior author (W. Zhang). Postoperative follow-up evaluations and management were performed by the intermediate authors. The review of medical records and the data collection and analyses were performed by the first author and research assistants.

We recorded information on the patients' operative time, successful tympanic membrane healing, postoperative complications, and their pre- and postoperative air conduction pure tone average (PTA), bone conduction PTA, and air-bone gap (ABG).

\section{Surgical procedures}

All patients underwent general anesthesia with endotracheal intubation. In the TEES group, endoscopic type I tragal cartilage tympanoplasty was performed with a fullthickness graft. The tragal cartilage perichondrium graft was harvested from the tragus, leaving a 2 - to $3-\mathrm{mm}$ rim at the dome of the cartilage to prevent deformation of the remaining tragus. The perichondrium was removed from the convex side of the tragal cartilage and an island graft was prepared, with the perichondrium being about 1-2 $\mathrm{mm}$ wider than the cartilage. A wedge-shaped incision was made from the center of the cartilage to accommodate the malleolus handle (Fig. 1a-f). Patients in the MES group underwent a post-auricular approach primary type I tympanoplasty that involved harvesting of graft tissue from the temporalis muscle facia. The graft materials were placed using an underlay technique medial to the remnant tympanic membrane. The external auditory canal and middle ear space were packed using gel foam.

\section{Outcome measures}

The main outcome measures were the surgical outcomes, restoration of hearing, and use of medical resources. The surgical outcomes included the successful graft rate and any postoperative complications. The restoration of hearing included pre- and postoperative ABG and average hearing gain (ABG improvement) in decibels. Use of medical resources included the average time spent in surgery in minutes.

External ear canal packing was removed within 3 weeks. Patients received regular endoscope and audiometric evaluation every 3 months for the first postoperative year and then on a yearly basis. A successful graft was defined as full healing of the grafted tympanic membrane, without perforation and retraction, for at least 6 months postoperatively. Audiometric data included preoperative and postoperative air conduction and bone conduction PTAs at each of 4 tested frequencies $(500,1000,2000$, and $4000 \mathrm{~Hz}$ ). The ABG was calculated from this information. Postoperative outcomes were calculated from the most recent audiogram available. 

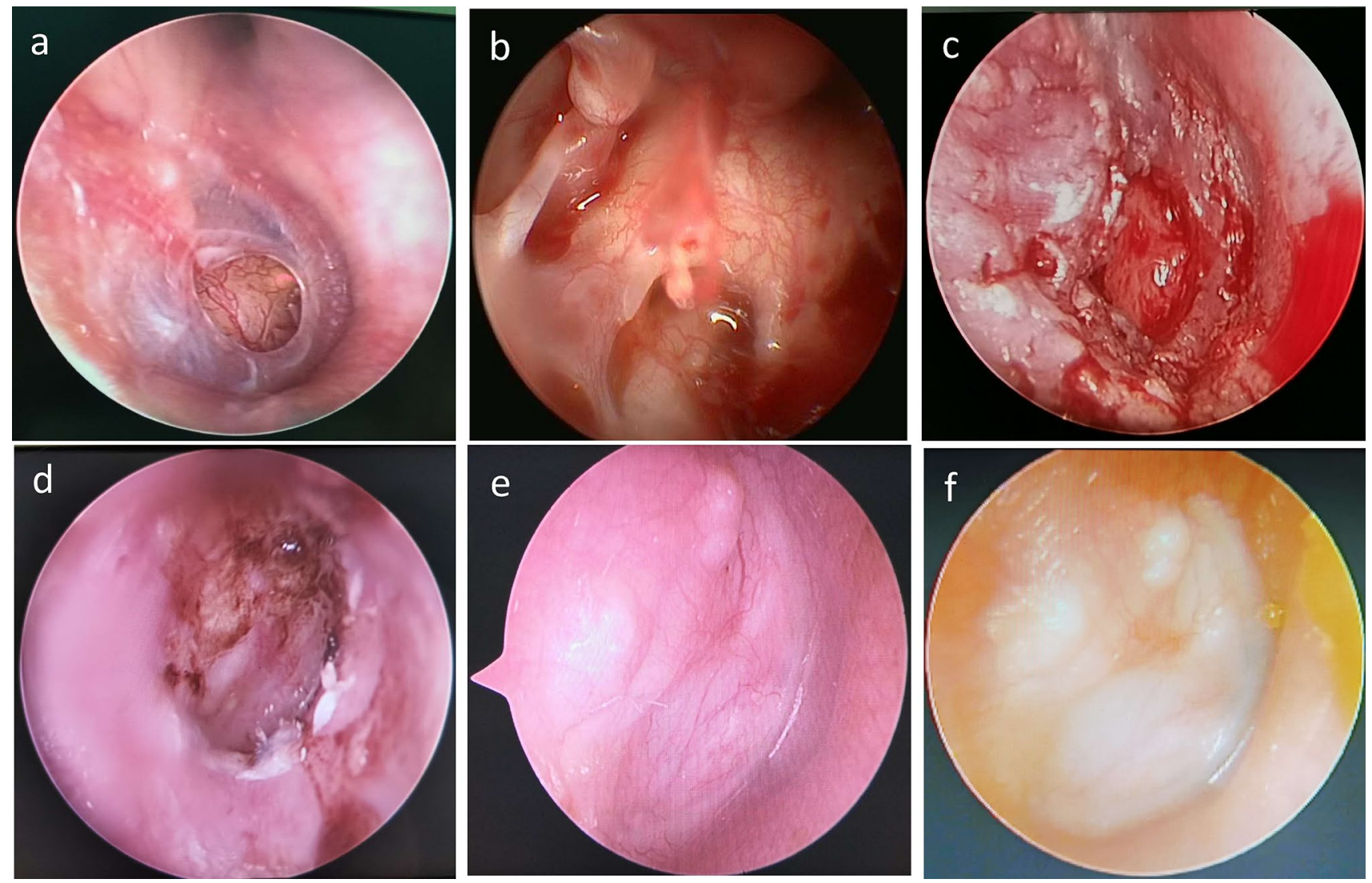

Fig. 1 Surgical steps and outcomes of TEES images (right era, $\mathbf{a}-\mathbf{f}$ ). Subtotal perforation of the ear drum (a). Visualization of middle ear by TEES (b). The tragal perichondrium graft was placed using an underlay technique (c). 1 month postoperative (d). 3 months postoperative (e). 6 months postoperative (f)

\section{Statistical analysis}

Data analysis was used SPSS version 23.0. Statistical comparisons were made using the chi-square test, and $t$ tests for independent samples. The results were assessed within a $95 \%$ reliance and at a significance level of $P<0.05$.

\section{Results}

\section{Clinical feature}

224 patients (100 female, 124 male) underwent endoscopic Type I tragal cartilage tympanoplasty, while 121 patients (56 female, 65 male) underwent microscopic tympanoplasty with temporalis facia. The mean \pm standard deviation (SD) age was $40.87 \pm 11.3$ years (range 18-67 years) in the endoscopic group and $38.56 \pm 11.5$ years (range 18-66 years) in the microscopic group; the difference in mean age was not statistically significant $(t=1.79 ; P=0.74)$ (Table 1$)$.

All patients were followed up for at least 6 months after surgical intervention. The mean \pm SD follow-up period was
Table 1 Baseline clinical and demographics of patients in two groups

\begin{tabular}{lll}
\hline & TEES $(n=224)$ & MES $(n=121)$ \\
\hline Average age \pm SD (years) & $40.87 \pm 11.3$ & $38.56 \pm 11.5$ \\
Sex & & \\
Male & 124 & 65 \\
Female & 100 & 56 \\
Lesion side & & \\
Left ear & 102 & 67 \\
$\quad$ Right ear & 122 & 54 \\
Average follow-up period \pm SD & $12.74 \pm 7.56$ & $14.08 \pm 6.36$ \\
$\quad$ (months) & & \\
Approach & & 0 \\
$\quad$ Transcanal & 224 & 0 \\
Endaural & 0 & 121 \\
Post-auricular & 0 & 0 \\
Graft material & & 121 \\
$\quad$ Tragal cartilage perichondrium & 224 & \\
$\quad$ graft & & \\
Temporalis muscle facia & 0 &
\end{tabular}


$12.74 \pm 7.56$ months (range 6-48 months)in the endoscopic group and $14.08 \pm 6.36$ months (range 6-36 months) in the microscopic group; the difference in mean follow-up was not statistically significant $(t=-1.66 ; P=0.098)$ (Table 1$)$.

\section{Comparison of surgical outcomes}

The mean $\pm \mathrm{SD}$ operative time was $49.22 \pm 8.24 \mathrm{~min}$ (range $30-70 \mathrm{~min}$ ) in the TEES group and $81.22 \pm 14.73 \mathrm{~min}$ (range $60-110 \mathrm{~min}$ ) in the MES group; the difference in mean operative time was statistically significant $(t=25.88 ; P<0.0001$; Table 2).

The successful graft rates of the TEES group at 1,3, and 6 (and > 6) months postoperatively were $97.77 \%, 96.88 \%$, and $94.64 \%$, which were better than the rates in the MES group, which were $97.5 \%, 95.87 \%$, and $90.90 \%$, respectively. However, the differences were not statistically significant $(P>0.05$; Table 2).

We analyzed the incidence of postoperative complications (Table 2). Patients in the MES group had a significant higher incidence of wound problems (ear pain, numbness around the ears, and wet ear; $P<0.05)$. However, there were no significant differences in tinnitus and abnormal taste. None of the postoperative patients experienced severe hearing loss or facial paralysis.

\section{Comparison of hearing outcomes}

Preoperatively, the air conduction levels of the operation ear were $37.846 \pm 9.41 \mathrm{~dB}$ in the TEES group and $36.508 \pm 9.83 \mathrm{~dB}$ in the MES group. There were no significant differences between the two groups $(P=0.216)$. The ABGs were $19.26 \pm 6.43 \mathrm{~dB}$ and $18.13 \pm 6.01 \mathrm{~dB}$, respectively. There were no statistically significant differences between the two groups $(P=0.113)$.

Table 2 Outcomes of group TEES and MES

\begin{tabular}{llll}
\hline & Group & \multirow{2}{l}{$P$} \\
\cline { 2 - 3 } & TEES $(n=224)$ & MES $(n=121)$ & \\
\hline Operative time & $49.22 \pm 8.24$ & $81.22 \pm 14.73$ & 0.000 \\
Tympanic membrane healing & & \\
Postoperative 1 month & $219(97.77 \%)$ & $118(97.5 \%)$ & 0.884 \\
Postoperative 3 months & $217(96.88 \%)$ & $116(95.87 \%)$ & 0.759 \\
Postoperative $\geq 6$ months & $212(94.64 \%)$ & $110(90.90 \%)$ & 0.239 \\
Postoperative complication & & & \\
Ear pain & $22(9.82 \%)$ & $101(83.47 \%)$ & 0.000 \\
Numbness around the & 0 & $24(19.83 \%)$ & 0.000 \\
$\quad$ ears & & & \\
Tinnitus & $5(2.23 \%)$ & $8(6.61 \%)$ & 0.071 \\
Wet ear & 0 & $5(4.13 \%)$ & 0.005 \\
Abnormal taste & $3(1.34 \%)$ & $2(1.65 \%)$ & 1.000 \\
\hline
\end{tabular}

Postoperatively, the improvements in the postoperative air conduction levels between the 2 groups at 1 month $(26.10 \pm 7.21 \mathrm{~dB}$ and $26.24 \pm 7.39 \mathrm{~dB}, P=0.87), 3$ months $(26.02 \pm 7.29 \mathrm{~dB}$ and $26.02 \pm 7.33 \mathrm{~dB}, P=0.999)$, and 6 (and > 6) months $(25.94 \pm 7.07 \mathrm{~dB}$ and $26.50 \pm 7.82 \mathrm{~dB}$; $P=0.427$ ) were not significantly different. The improvements in the postoperative ABGs between the 2 groups were not significantly different at 1 month $(7.85 \pm 4.14 \mathrm{~dB}$ and $8.02 \pm 4.26 \mathrm{~dB} ; P=0.727), 3$ months $(7.75 \pm 4.11 \mathrm{~dB}$ and $7.94 \pm 4.26 \mathrm{~dB} ; P=0.681$ ), and 6 (and $>6$ ) months $(7.72 \pm 4.00 \mathrm{~dB}$ and $8.34 \pm 4.84 \mathrm{~dB}, P=0.201)$. The average hearing gain in the TEES group at 6 (and $>6$ ) months postoperatively was $11.85 \pm 5.47 \mathrm{~dB}$, which was better than $10.48 \pm 5.18 \mathrm{~dB}$ in the MES group $(P=0.031)$. There were no statistically significant differences between the 2 groups at 1 and 3 months postoperatively for average hearing gains (Table 3).

\section{Discussion}

The goals of tympanoplasty are reconstruction of a health tympanic cavity, closure of the perforation, and optimal restoration of hearing [9]. MES using a post-auricular approach or endaural approach is a traditional and commonly used tympanoplasty technique, with success rates ranging from 83 to $100 \%$ [10-16].

Table 3 Pre- and post-operative hearing conditions of two groups

\begin{tabular}{llll}
\hline & Group & \multirow{2}{*}{$P$} \\
\cline { 2 - 3 } & TEES $(n=224)$ & MES $(n=121)$ & \\
\hline Air condition (dB) & & & \\
Preoperative & $37.846 \pm 9.41$ & $36.508 \pm 9.83$ & 0.216 \\
Postoperative 1 month & $26.10 \pm 7.21$ & $26.24 \pm 7.39$ & 0.87 \\
Postoperative 3 months & $26.02 \pm 7.29$ & $26.02 \pm 7.33$ & 0.999 \\
Postoperative $\geq 6$ months & $25.94 \pm 7.07$ & $26.50 \pm 7.82$ & 0.427 \\
Bone-condition (dB) & & & \\
Preoperative & $18.69 \pm 1.70$ & $18.38 \pm 5.29$ & 0.588 \\
Postoperative 1 month & $18.25 \pm 4.61$ & $18.22 \pm 4.74$ & 0.954 \\
Postoperative 3 months & $18.27 \pm 4.62$ & $18.24 \pm 4.75$ & 0.343 \\
Postoperative $\geq 6$ months & $18.21 \pm 4.57$ & $18.25 \pm 4.74$ & 0.948 \\
Air-bone gap (dB) & & & \\
Preoperative & $19.26 \pm 6.43$ & $18.13 \pm 6.01$ & 0.113 \\
Postoperative 1 month & $7.85 \pm 4.14$ & $8.02 \pm 4.26$ & 0.727 \\
Postoperative 3 months & $7.75 \pm 4.11$ & $7.94 \pm 4.26$ & 0.681 \\
Postoperative $\geq 6$ months & $7.72 \pm 4.00$ & $8.34 \pm 4.84$ & 0.201 \\
Average hearing gain (dB) & & & \\
Postoperative 1 month & $11.61 \pm 5.55$ & $10.40 \pm 5.18$ & 0.052 \\
Postoperative 3 months & $11.76 \pm 5.43$ & $10.71 \pm 5.31$ & 0.091 \\
Postoperative $\geq 6$ months & $11.85 \pm 5.47$ & $10.48 \pm 5.18$ & 0.031 \\
\hline
\end{tabular}


However, with the straight-line vision of microscopes, the surgeon cannot visualize the middle ear through the ear canal. As a result, MES approaches frequently necessitate soft tissue retraction, with or without drilling of the bone, to visualize the diseased area satisfactorily [17, 18]. This may prolong the operative time and increase the incidence of postoperative complications $[18,19]$. In this study, we observed the mean operative time was significantly longer in the MES group than in the TEES group $(t=25.88 ; P<0.0001)$. Patients receiving MES had a significantly higher incidence of wound problems (ear pain, numbness around the ears, and wet ear; $P<0.05$ ). In a study by Huang et al. [20], the mean operative time was 50.4 min among 50 patients who underwent endoscopic tympanoplasty, compared with $75.5 \mathrm{~min}$ for the microscopic approach $(P<0.0001)$. Hsu et al. [21] also reported that the mean duration of surgery and the operative time were shorter in the TEES group than in the MES group, and the postoperative complications (severe sensorineural hearing loss, mastoiditis, and persistent otorrhea) were less common. Choi et al. [16] also reported that patients who underwent TEES experienced significantly less pain at the first day after surgery than patients who underwent MES.

We infer that TEES transforms the external ear canal into an operative area [22, 23], leaving far less soft-tissue damage, no hair loss, and only a tiny wound associated with graft harvesting [24, 25], which may somewhat influence the need for hospitalization [26]. Previous studies $[13,27,28]$ used endoscopes instead of microscopes for tympanoplasty and cholesteatoma surgery and noted the following advantages of TEES. Specifically, TEES can provide good illumination, shorten the focal length of the observation object, expand the viewing angle, and explore the "blind area" under the microscope, with minimal interference in the middle tympanum $[24,25]$.

In our study, the perforation closure rates were achieved in 219 patients (97.77\%) in the TEES group and in 118 patients (97.5\%) in the MES group at 1 month post surgery. The perforation closure rates were similar in the TEES and MES group with a postoperative follow-up period of at least 6 months $(94.64 \%$ vs $90.9 \%, P=0.239)$. Hsu et al. [21] also reported no significant differences in the graft success rates of in TEES and MES groups (96.2\% vs $92.0 \%, P=0.2826$ ) of patients who underwent tympanoplasty without cholesteatoma. Ohki et al. [29] reported on 47 patients who underwent TEES without ossiculoplasty for chronic otitis media with a 1-year follow-up period. The surgical success rates for tympanic membrane closure were $93.6 \%$ for the TEES group and $85.3 \%$ for the postauricular MES group $(P=0.244)$. The surgical success rate for hearing (air-bone gap $\leq 20 \mathrm{~dB}$ ) was $95.7 \%$ in the TEES group and $84.0 \%$ in the post-auricular MES group $(P=0.077)$.
These outcomes are consistent with those obtained in our study. The endoscopic approach is less invasive (no skin incisions or canalplasty are required), results in a good graft success rate, and enables better visualization. Compared with the microscopic approach, the endoscopic approach can reduce postoperative bleeding and pain and achieve superior cosmetic results [30]. In addition, minimal damage to healthy structures is likely to minimize postoperative adverse reactions locally and generally.

Both groups in the current study had equal improvements in hearing and ABG. There were no statistically significant differences in the preoperative ABG $(\mathrm{dB})(P=0.113)$ and postoperative $\mathrm{ABG}(\mathrm{dB})$ at 1 month $(P=0.727)$, 3 months $(P=0.681)$, and 6 months $(P=0.201)$ between the TEES and MES groups in hearing restoration. We also observed the average postoperative hearing gain $(\mathrm{dB})$ at 1 and 3 months were not significantly different between the 2 groups (Table 3). However, the average postoperative hearing gains at 6 (and $>6$ ) months were better in the TEES group than in the MES group $(11.85 \pm 5.47 \mathrm{~dB}$ vs $10.48 \pm 5.18 \mathrm{~dB}, P=0.031)$. We assume that the graft source is the reason for this difference because the elasticity of cartilage perichondrium may be superior to that of temporal muscle fascia. However, this result is limited due to the lack of long-term outcomes. Onal et al. [31] evaluated primary tympanoplasty cases with a subtotal perforation to compare the functional results of type I tympanoplasty in patients with bilateral chronic otitis media. The graft success rate for at least 12 months postoperatively was $65.9 \%$ for the fascia group and $92.3 \%$ for the cartilage group. There was no statistically significant difference in postoperative hearing gain between the two groups $(11.63 \pm 12.62 \mathrm{~dB}$ in the fascia group vs $14.49 \pm 10.05 \mathrm{~dB}$ in the cartilage group, $P=0.268$ ). It was reported that there were no statistically significant differences in the preoperative $(21.6 \pm 11.2 \mathrm{~dB}$ versus $21.4 \pm 10.6 \mathrm{~dB}, P=0.9507)$ and postoperative ( $>6$ months) ABG $(8.90 \pm 10.0 \mathrm{~dB}$ versus $8.3 \pm 10.0 \mathrm{~dB}, P=0.7641)$ between the TEES and MES groups in hearing restoration. Hsu et al. [21] also reported that the average hearing gains following surgery were $10.27 \pm 6.4 \mathrm{~dB}$ and $12.43 \pm 7.46 \mathrm{~dB}$ in TEES and MES $(P=0.1663)$.

In our series, we achieved a graft success rate of $94.64 \%$ following an endoscopic tympanoplasty at least 6 months, with satisfying improvements in hearing and fewer postoperative complications. To reduce selection bias by adopting TEES and MES in a consecutive series in this study, we selected a clear temporal division (before and since 2013) rather than one based on disease severity. The basic characteristics of the TEES and MES groups were compared, including potential risk factors such as sex and age of the patient, size of the perforation, primary operation, status of the middle ear mucosa, preoperative hearing level, and preoperative air-bone gap. There was no significant 
difference in any of the parameters. All of the operations in the present study were performed by a single surgeon (W. Zhang) who has been using fascia and cartilage grafts for tympanoplasty for more than 20 years. Thus, we eliminated between surgeon variations in approach as a confounding factor. Although some confounding factors exist, such as different grafts materials and part of the patients in the TEES group were within the learning curve, the results of our study reflect clinical reality to some extent. Therefore, we believed that TEES could achieve surgical outcomes as good as those of MES and reduce postoperative complications and use of medical resources due to a shorter procedure time.

\section{Limitation}

This retrospective study was limited by a number of factors. First, we did not report the other grafting materials in TEES because the data were less and incomplete. Second, MES via endaural approach was not mentioned throughout the study because all of the MES in our hospital were performed by the senior author (W. Zhang) via the postauricular approach. Finally, this study conducted at a single hospital. A more extensive survey of cases a relatively long-term follow-up results or a multi-hospital study would be beneficial.

\section{Conclusion}

Under favorable conditions, TEES and MES produced comparable hearing outcomes. TEES was a more beneficial approach for hearing gain than MES. With respect to tympanic membrane repair, TEES and MES were comparably effective. However, prospective studies should be conducted in future to confirm these conclusions.

Acknowledgements The authors thank the help from the central laboratory of Shaanxi Provincial People's Hospital.

Author contributions QM Yang oversaw data collection and verification, was responsible interpretation of results, and writing of the manuscript. BW and JZ was responsible for data collection and interpretation of results. MX and HL were responsible for the statistical concept and analysis. WZ were responsible for the study conception, data interpretation and made major contributions to the manuscript. All authors have read and approved the final version of the manuscript.

Funding The study was funded by the research funds of Shaanxi Province Key Research and Development plan project (No:2018ZDXM-SF-048)

\section{Declarations}

Conflict of interest No conflict of interest was declared by the authors.
Open Access This article is licensed under a Creative Commons Attribution 4.0 International License, which permits use, sharing, adaptation, distribution and reproduction in any medium or format, as long as you give appropriate credit to the original author(s) and the source, provide a link to the Creative Commons licence, and indicate if changes were made. The images or other third party material in this article are included in the article's Creative Commons licence, unless indicated otherwise in a credit line to the material. If material is not included in the article's Creative Commons licence and your intended use is not permitted by statutory regulation or exceeds the permitted use, you will need to obtain permission directly from the copyright holder. To view a copy of this licence, visit http://creativecommons.org/licenses/by/4.0/.

\section{References}

1. Schwam ZG, Cosetti MK (2021) Endoscopic myringoplasty and type I tympanoplasty. Otolaryngol Clin N Am 54(1):75-88

2. Salvador P, Gomes P, Silva F, Fonseca R (2021) Type I tympanoplasty: surgical success and prognostic factors. Acta Otorrinolaringol Esp (Engl Ed) 72(3):182-189

3. Marchioni D, Gazzini L, De Rossi S, Di Maro F, Sacchetto L, Carner M, Bianconi L (2020) The management of tympanic membrane perforation with endoscopic type I tympanoplasty. Otol Neurotol 41(2):214-221

4. Thomassin JM, Duchon-Doris JM, Emram B, Rud C, Conciatori J, Vilcoq P (1990) Endoscopic ear surgery. Initial evaluation. Ann Otolaryngol Chir Cervicofac 107(8):564-570

5. Mittermiller PA, Rochlin DH, Menard RM (2020) Endoscopic spring-mediated distraction for unilambdoid craniosynostosis. J Craniofac Surg 31(7):2097-2100

6. Zhao C, Ji Y, An Y, Xue J, Li Q, Suo L, Hou R, Zhang Y, Geng Z, Shen $\mathrm{H}$ et al (2018) An alternative method of endoscopic intrasphenoidal vidian neurectomy. OTO Open 2(1):2473974X-18764862X

7. Emre IE, Cingi C, Bayar MN, Nogueira JF (2020) Endoscopic ear surgery. J Otol 15(1):27-32

8. Mitchell S, Coulson C (2017) Endoscopic ear surgery: a hot topic? J Laryngol Otol 131(2):117-122

9. Alicandri-Ciufelli M, Marchioni D, Grammatica A, Soloperto D, Carpeggiani P, Monzani D, Presutti L (2012) Tympanoplasty: an up-to-date pictorial review. J Neuroradiol 39(3):149-157

10. Dornhoffer JL (1997) Hearing results with cartilage tympanoplasty. Laryngoscope 107:1094-1099

11. Indorewala S, Adedeji TO, Indorewala A, Nemade G (2015) Tympanoplasty outcomes: a review of 789 cases. Iran J Otorhinolaryngol 27:101-108

12. Kumar M, Kanaujia S, Singh A (2015) A comparative study of endoscopic myringoplasty vs conventional myringoplasty. Otorhinolaryngol Clin Int J 7:132-137

13. Kaya I, Sezgin B, Sergin D, Ozturk A, Eraslan S, Gode S, Bilgen C, Kirazli T (2017) Endoscopic versus microscopic type 1 tympanoplasty in the same patients: a prospective randomized controlled trial. Eur Arch Otorhinolaryngol 274(9):3343-3349

14. El-Hennawi DEM, Ahmed MR, Abou-Halawa AS, Al-Hamtary MA (2018) Endoscopic push-through technique compared to microscopic underlay myringoplasty in anterior tympanic membrane perforations. J Laryngol Otol 132:509-513

15. Dundar R, Kulduk E, Soy FK et al (2014) Endoscopic versus microscopic approach to type 1 tympanoplasty in children. Int $\mathrm{J}$ Pediatr Otorhinolaryngol 78:1084-1089

16. Choi N, Noh Y, Park W et al (2017) Comparison of endoscopic tympanoplasty to microscopic tympanoplasty. Clin Exp Otorhinolaryngol 10:44-49

Sponsorships None. 
17. Han SY, Lee DY, Chung J, Kim YH (2019) Comparison of endoscopic and microscopic ear surgery in pediatric patients: a metaanalysis. Laryngoscope 129(6): 1444-1452

18. Kozin ED, Gulati S, Kaplan AB, Lehmann AE, Remenschneider AK, Landegger LD, Cohen MS, Lee DJ (2015) Systematic review of outcomes following observational and operative endoscopic middle ear surgery. Laryngoscope 125(5):1205-1214

19. Bae MR, Kang WS, Chung JW (2019) Comparison of the clinical results of attic cholesteatoma treatment: endoscopic versus microscopic ear surgery. Clin Exp Otorhinolaryngol 12(2):156-162

20. Huang TY, Ho KY, Wang LF, Chien CY, Wang HM (2016) A comparative study of endoscopic and microscopic approach type 1 tympanoplasty for simple chronic otitis media. J Int Adv Otol 12(1):28-31

21. Hsu YC, Kuo CL, Huang TC (2018) A retrospective comparative study of endoscopic and microscopic Tympanoplasty. J Otolaryngol Head Neck Surg 47(1):44

22. Plodpai Y, Paje N (2017) The outcomes of overlay myringoplasty: endoscopic versus microscopic approach. Am J Otolaryngol 38(5):542-546

23. Ji L, Zhai S (2020) Comparison of frequency-specific hearing outcomes after endoscopic and microscopic tympanoplasty. Acta Otolaryngol 140(12):990-994

24. Kim MS, Chung J, Kang JY, Choi JW (2020) Transcanal endoscopic ear surgery for traumatic ossicular injury. Acta Otolaryngol 140(1):22-26

25. Ito T, Kubota T, Furukawa T, Matsui H, Futai K, Kakehata S (2019) Transcanal endoscopic ear surgery for congenital middle ear anomalies. Otol Neurotol 40(10):1299-1305
26. Glikson E, Yousovich R, Mansour J, Wolf M, Migirov L, Shapira Y (2017) Transcanal endoscopic ear surgery for middle ear cholesteatoma. Otol Neurotol 38(5):e41-e45

27. Nassif N, Berlucchi M, Redaelli DZL (2015) Tympanic membrane perforation in children: endoscopic type I tympanoplasty, a newly technique, is it worthwhile? Int J Pediatr Otorhinolaryngol 79(11):1860-1864

28. Lakpathi G, Sudarshan RL, Anand (2016) Comparative study of endoscope assisted myringoplasty and microscopic myringoplasty. Indian J Otolaryngol Head Neck Surg 68(2):185-190

29. Ohki M, Kikuchi S, Tanaka S (2019) Endoscopic type 1 tympanoplasty in chronic otitis media: comparative study with a postauricular microscopic approach. Otolaryngol Head Neck Surg 161(2):315-323

30. Mundra RK, Sinha R, Agrawal R (2013) Tympanoplasty in subtotal perforation with graft supported by a slice of cartilage: a study with near $100 \%$ results. Indian J Otolaryngol Head Neck Surg 65(Suppl 3):631-635

31. Onal K, Arslanoglu S, Songu M, Demiray U, Demirpehlivan IA (2012) Functional results of temporalis fascia versus cartilage tympanoplasty in patients with bilateral chronic otitis media. J Laryngol Otol 126(1):22-25

Publisher's Note Springer Nature remains neutral with regard to jurisdictional claims in published maps and institutional affiliations. 